\title{
Avaliação da idade dentária em crianças e adolescentes com epidermólise bolhosa - série de casos
}

\author{
Dental age estimation in children and adolescents with epidermolysis bullosa \\ - case series
}

\author{
Lorena Vieira Santos $^{1 *}$, Patrícia Leite Ribeiro ${ }^{2}$ \\ ${ }^{1}$ Cirurgiã-dentista. Mestranda do Programa de Pós-graduação Processos Interativos dos Órgãos e Sistemas. Instituto \\ de Ciências da Saúde. Universidade Federal da Bahia - UFBA; ${ }^{2}$ Doutora em Radiologia Odontológica. Professora \\ Associada. Faculdade de Odontologia - UFBA
}

\begin{abstract}
Resumo
Introdução: a epidermólise bolhosa compreende um grupo de doenças genéticas e raras da pele, onde há uma fragilidade estrutural da pele e das mucosas. A gravidade das manifestações clínicas varia de acordo com o subtipo envolvido, no qual a citada enfermidade distrófica recessiva é a mais severa. Há várias implicações sistêmicas, como a anemia, a desidratação e a desnutrição. O envolvimento bucal acompanha e torna mais severo o comprometimento sistêmico. As alterações em tecidos moles e duros dessa região variam consideravelmente. São relatados atrasos no crescimento e desenvolvimento dentoesquelético. Objetivo: considerando as possíveis alterações de desenvolvimento, este estudo tem o objetivo de mensurar a idade dentária em pacientes com epidermólise bolhosa distrófica recessiva, verificando a acurácia de dois métodos em relação à idade cronológica. Metodologia: foi realizado um estudo descritivo analítico transversal do tipo série de casos, que avaliou a idade dentária de 10 pacientes pelos métodos de Demirjian e Willems. Resultados: não houve divergência significativa entre as idades dentária e cronológica, com superestimação comum aos métodos utilizados. Conclusão: sugere-se que o tempo de maturação dentária pode ser um parâmetro estável frente a fatores adversos ao desenvolvimento, que são comuns a esse grupo. São necessários estudos que avaliem também outras variáveis para melhor compreender a relação da epidermólise bolhosa com o desenvolvimento dentoesquelético.

Palavras-chave: Epidermólise bolhosa. Determinação da idade pelos dentes. Desenvolvimento Infantil.
\end{abstract}

\begin{abstract}
Introduction: Epidermolysis Bullosa (EB) describes a group of rare genetic skin diseases where there is structural fragility within the skin and mucosa. Severity of clinical manifestations varies according to the specific EB type, and the most severe EB type is recessive dystrophic $E B$. There are several systemic implications, such as anemia, dehydration and malnutrition. There are also oral manifestations, so systemic manifestations may be more severe. Changes in soft and hard oral tissues vary considerably. Dentofacial growth and development retardation is reported. Aim: considering developmental changes, this study aims to measure the dental age of patients with recessive dystrophic EB by verifying the accuracy of two methods in relation to chronological age. Methods: a descriptive cross-sectional and analytical study of case series was carried out, which assessed the dental age of 10 patients using Demirjian and Willems methods. Results: there was not significant divergence between dental and chronological ages, but there was mutual overestimation related to both methods. Conclusion: it is suggested that dental maturation period may be a stable parameter against adverse developmental factors that are common to this group. Studies that also assess other variables are necessary to better understand the relation between EB and dentofacial growth.

Keywords: Epidermolysis bullosa. Age determination by teeth. Child development.
\end{abstract}

\section{INTRODUÇÃO}

A epidermólise bolhosa (EB) compreende um grupo de doenças genéticas e raras, de natureza hereditária, na qual há uma fragilidade estrutural da pele e mucosas, manifestada desde os primeiros dias de vida (BRASIL. MINISTÉRIO DA SAÚDE, 2019). Mutações das proteínas da junção dermoepidérmica determinam a formação de bolhas de forma espontânea ou ao mínimo trauma, que facilmente se rompem e tornam-se úlceras, muitas

Correspondente/Correspoding: *Lorena Vieira Santos - End: Rua Cruzador Bahia, $\mathrm{n} \cong 4$, Nazaré, Salvador, Bahia, CEP: 40040-505. Salvador BA - Tel: (71) 99187-4419-E-mail: Lorenavs@ufba.br vezes dolorosas. Deve-se diferenciar desse grupo a EB adquirida, de natureza autoimune e que se inicia em outras faixas etárias. Não há dados epidemiológicos sobre a frequência da EB no Brasil (BRASIL. MINISTÉRIO DA SAÚDE, 2019) em associação à ausência de notificação compulsória das doenças raras no país.

A EB apresenta transmissão autossômica dominante ou recessiva e se classifica nos tipos simples, juncional, distrófica e síndrome de Kindler (BRASIL. MINISTÉRIO DA SAÚDE, 2019; KRÄMER et al., 2020). A classificação varia conforme o nível de clivagem da pele, os genes e as proteínas-alvo envolvidos. As subclassificações consideram ainda as características fenotípicas clínicas, como 
distribuição (localizada ou generalizada) e gravidade relativa quanto ao envolvimento cutâneo e extracutâneo (KRÄMER et al., 2020).

A severidade das manifestações clínicas varia de acordo com o subtipo envolvido. Destaca-se a pseudossindactilia, decorrente das bolhas repetidas e cicatrizes nas mãos e nos pés, que traz limitações funcionais/ motoras, comuns da EB distrófica recessiva, associada a repercussões mais graves da doença (COHN; TENG, 2016). Há várias implicações sistêmicas, como anemia, desidratação e desnutrição (KRÄMER et al., 2020; PITTA; MAGALHÃES; SILVA, 2016; ZIDORIO et al., 2015). Além disso, a redução da atividade física, menor exposição solar, uso de corticoides e deficit nutricional, comum a esses pacientes, potencialmente afetam saúde óssea (RODARI et al., 2017).

O envolvimento bucal acompanha e potencializa o comprometimento sistêmico. As alterações em tecidos moles e duros dessa região variam consideravelmente. São relatados atrasos no crescimento e no desenvolvimento dentário e esquelético, além de hipodontia e hipoplasia do esmalte (COHN; TENG, 2016). As repetidas lesões e o consequente tecido cicatricial promovem a formação de deformidades na arquitetura bucal, como a microstomia, perda de fundo de vestíbulo e anquiloglossia, características comuns na EB distrófica recessiva (COHN; TENG, 2016). Essas deformidades podem alterar a função muscular e resultar em deficiências dimensionais na maxila e na mandíbula e em alterações nas arcadas dentárias (NAVA; ÁNGELES; DURAN, 2014). Além disso, a presença das lesões dolorosas compromete a alimentação e a higiene intrabucal, gerando os impactos nutricionais e infecciosos (ZIDORIO et al., 2015), tornando importantes a assistência odontológica e os cuidados especiais nesses pacientes (KRÄMER et al., 2020).

Considerando as possíveis alterações de desenvolvimento, o objetivo deste estudo é mensurar a idade dentária de 10 pacientes com EB distrófica recessiva, verificando a acurácia de dois métodos em relação à idade cronológica.

\section{METODOLOGIA}

Este é um estudo descritivo analítico transversal, do tipo série de casos, autorizado pelo Comitê de Ética em Pesquisa do Complexo Hospitalar Professor Edgard Santos (complexo HUPES), da Universidade Federal da Bahia.

Por se tratar de uma doença rara, foi utilizada uma amostra de conveniência composta por pacientes atendidos pelo serviço de Odontologia do complexo HUPES, que realizaram TC da face ou radiografia panorâmica na faixa etária de 3 a 17 anos.

A coleta de dados foi realizada por meio de consulta em prontuário eletrônico e físico, no ano de 2020. Incluíram-se neste estudo os pacientes que tinham acompanhamento no serviço de Odontologia do HUPES, diagnosticados com EB distrófica recessiva, e que contavam com radiografia panorâmica ou TC de face.

Os exames de imagem foram analisados por um examinador treinado, comparando-as imagens com o diagrama dos estágios de desenvolvimento dentário e as características descritas para cada estágio de acordo com a classificação de Demirjian (DEMIRJIAN; GOLDSTEIN; TANNER, 1973). Avaliaram-se os sete dentes permanentes inferiores do lado esquerdo $(37,36,35$, 34, 33, 32 e 31) de cada paciente. Em caso de ausência dentária, foi consultado o dente correspondente no hemiarco inferior direito. Em caso de ausência do dente correspondente foi selecionado o dente antagonista. As idades dentárias foram obtidas através das tabelas de Demirjian e de Willems (DEMIRJIAN; GOLDSTEIN; TANNER, 1973; WILLEMS et al., 2001). Ambos métodos utilizam o mesmo diagrama para classificar o estágio de maturação. A partir dessa classificação obtiveram-se pontuações correspondentes ao estágio de cada dente que foram somadas, de acordo com as tabelas supracitadas. Através do método de Demirjian, foi necessário converter o valor obtido dessa soma em anos, enquanto que pelo método de Willens foi necessário apenas somar os valores que já estavam convertidos em anos.

A tabulação dos dados foi estruturada uma planilha em Excel, com os seguintes tópicos: identificação do paciente, sexo, idade cronológica, idade dentária de acordo cada método e diferença entre idade cronológica e dentária. A idade cronológica foi calculada automaticamente na planilha por meio de uma fórmula, obtendo-se a idade em meses e dividindo-a por 12. Além disso, foram obtidos a média aritmética e o desvio padrão da idade cronológica e das idades estimadas e realizou-se o teste de Bland-Altman para verificar a acurácia das idades estimadas em relação à cronológica, obtendo-se visualização gráfica pelo pacote estatístico.

\section{RESULTADOS}

Foram acessados prontuários de 45 pacientes e selecionados 17 , contendo registro dos exames de imagem. Excluíram-se 7 pacientes que estavam fora da faixa etária requerida na data do exame, sem diagnóstico do subtipo distrófico recessivo ou sem acesso à radiografia realizada. Foram selecionados 10 exames de imagem, dos quais 4 foram tomografias e 6 , radiografias panorâmicas. Dentre os pacientes, 8 são do sexo masculino e 2 do sexo feminino, com idades cronológicas de 5 a 16 anos no momento do exame (Tabela 1 ).

Houve resultados diversos ao calcular a diferença entre as idades dentária e cronológica em cada paciente (Tabela 1). Pelo método de Demirjian, 7 pacientes apresentaram superestimação e 3 , subestimação da idade dentária. Pelo método de Willems, 5 apresentaram superestimação e 5, subestimação. Os valores variaram - 0,92 a 2,63 no primeiro método e $-0,89$ a 1,17 , com valores extremos menos distantes em Willems. Ao comparar a média das idades cronológicas e das idades dentárias, 
observa-se superestimação das idades dentárias em ambos os métodos neste grupo (Tabela 2). A acurácia das idades dentárias estimadas nos dois métodos em relação à idade cronológica (padrão-ouro) foi demonstrada através do gráfico de Bland-Altman, onde nota-se pequeno viés em relação à idade cronológica, apresentando valor negativo, correspondente à superestimação (Gráfico 1 e $2)$, com maior viés para Demerjian $(-0,71)$.

Tabela 1 - Estimativa da idade em pacientes com EB distrófica recessiva.

\begin{tabular}{|c|c|c|c|c|c|c|}
\hline \multirow[b]{3}{*}{ PACIENTE } & \multirow[b]{3}{*}{ SEXO } & \multirow[b]{3}{*}{ IC } & \multicolumn{4}{|c|}{ MÉTODO } \\
\hline & & & \multicolumn{2}{|c|}{ DEMIRJIAN } & \multicolumn{2}{|c|}{ WILLEMS } \\
\hline & & & ID & ID-IC & ID & ID-IC \\
\hline 1 & $\mathrm{~F}$ & 5,67 & 7,4 & 1,73 & 6,65 & 0,98 \\
\hline 2 & M & 6,17 & 7,7 & 1,53 & 7,24 & 1,07 \\
\hline 3 & M & 7,33 & 7,8 & 0,47 & 7,46 & 0,13 \\
\hline 4 & M & 8,00 & 7,8 & $-0,20$ & 7,4 & $-0,60$ \\
\hline 5 & $\mathrm{~F}$ & 11,25 & 11,6 & 0,35 & 10,67 & $-0,58$ \\
\hline 6 & M & 11,42 & 11,8 & 0,38 & 11,26 & $-0,16$ \\
\hline 7 & M & 11,58 & 10,9 & $-0,68$ & 11,01 & $-0,57$ \\
\hline 8 & M & 13,17 & 15,8 & 2,63 & 14,34 & 1,17 \\
\hline 9 & $M$ & 14,17 & 16 & 1,83 & 15,28 & 1,11 \\
\hline 10 & $M$ & 16,92 & 16 & $-0,92$ & 16,03 & $-0,89$ \\
\hline
\end{tabular}

Fonte: Dados da pesquisa.

Legenda: Idade cronológica (IC), Idade dentária (ID); valores em anos.

Tabela 2 - Média das estimativas das idades em pacientes com EB distrófica recessiva.

\begin{tabular}{lc}
\hline \multicolumn{1}{c}{ Características gerais* } & N = 10 \\
\hline $\begin{array}{l}\text { Idade cronológica (anos), Média (DP)(CV\%) } \\
\text { 1dade dentária Demirjian (anos), Média (DP) }\end{array}$ & $11,69)(34,9)$ \\
$\begin{array}{l}\text { (CV\%) } \\
\text { Idade dentária Willems (anos), Média (DP) }\end{array}$ & $132,0)$ \\
(CV\%) & $10,73(3,54)(32,9)$ \\
\hline
\end{tabular}

Fonte: Dados da pesquisa

Legenda: *DP: desvio-padrão; CV: coeficiente de variação da média \%
Gráfico 1 - Acurácia das idades estimadas no método de Demirjian em relação à cronológica (padrão-ouro) através do gráfico de Bland-Altman.

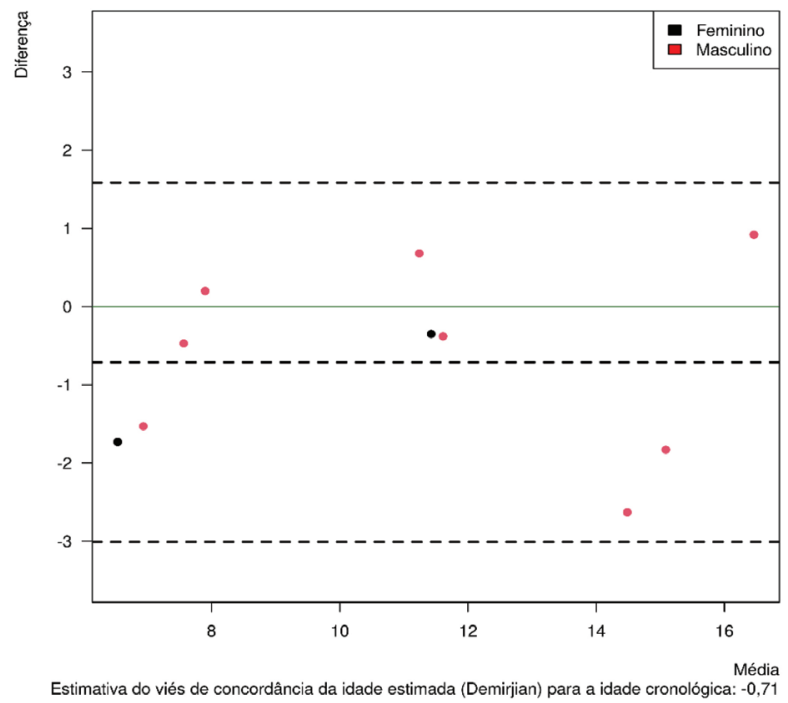

Fonte: Dados da pesquisa.

Gráfico 2-Acurácia das idades estimadas no método de Willems em relação à cronológica (padrão-ouro) através do gráfico de Bland-Altman.

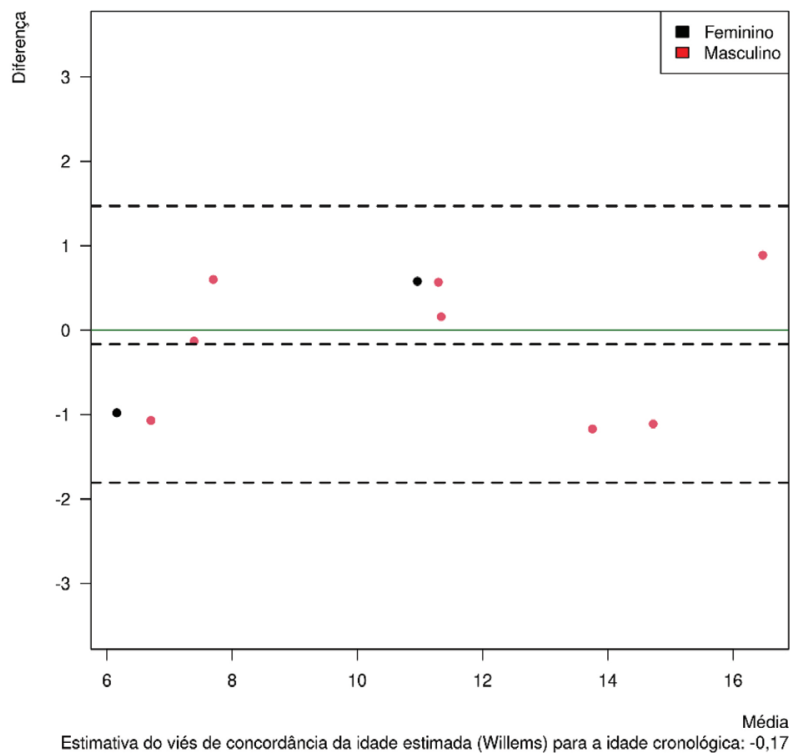

Fonte: Dados da pesquisa.

\section{DISCUSSÃO}

Os pacientes com EB distrófica recessiva apresentam uma condição sistêmica que pode levar a alterações e atrasos no seu desenvolvimento e crescimento geral (ZIDORIO et al., 2015). Há o comprometimento do sistema estomatognático à medida que há a interrelação de fatores como desnutrição, anemia, lesões e alterações morfofuncionais em tecidos moles, além de implicações dentárias e esqueléticas. 
A desnutrição energético-proteica durante a fase de desenvolvimento dentário se relaciona com uma maior suscetibilidade à cárie dentária, atraso na cronologia de irrupção e defeitos estruturais do esmalte, como hipoplasia do esmalte, além de hipofunção das glândulas salivares e mudanças na composição da saliva (COSTA et al., 2010; DIMAISIP-NABUAB et al., 2018). Ademais, alguns pacientes apresentam condição social desfavorável, o que proporciona impactos negativos sobre sua saúde e crescimento. Contudo, a relação causal entre fatores nutricionais, regionais, socioeconômicos e alterações no desenvolvimento e irrupção do dente, ainda é tema em debate e têm evidências pouco substanciais.

Este estudo considerou a hipótese de haver atraso no desenvolvimento dentário em pacientes com EB distrófica recessiva, devido às implicações sistêmicas secundárias a esta condição, como a desnutrição. Atrasos no desenvolvimento dentário podem ser um fator de risco para maloclusão, sobretudo quando há interferência na ordem cronológica de irrupção dentária. Em geral, atrasos de desenvolvimento podem ser medidos pela estimativa da idade biológica, com a análise da maturação de diversos tecidos, como o ósseo e o dentário. Essas análises também podem ser utilizadas para fins legais de estimativa da idade.

Para avaliação da idade dentária, é possível observar o padrão de calcificação do dente ou o processo de irrupção. Os métodos que avaliam a formação do dente são mais seguros, visto que o desenvolvimento dentário é um processo contínuo e menos influenciado por fatores intrínsecos e extrínsecos em comparação à irrupção (WILLEMS et al., 2001). Ainda assim, considera-se que ocorrem variações no tempo de desenvolvimento dentário, possivelmente influenciadas por fatores genéticos e ambientais (ESAN; YENGOPAL; SCHEPARTZ, 2017).

A avaliação da maturação dentária vem se mostrando um meio confiável para calcular a idade biológica dos indivíduos, mas não tanto para verificar os efeitos de alterações sistêmicas no desenvolvimento dentário. Para a estimativa da idade, autores enfatizam a superioridade de se avaliar o grau de mineralização dentária por ser menos afetado por fatores ambientais (CONCEIÇÃO; CARDOSO, 2011). As condições ambientais parecem afetar mais a idade óssea do que a dentária, mas são necessários mais estudos que elucidem se há isenção de associação com o desenvolvimento dentário.

Alguns estudos encontram relação positiva entre desenvolvimento ósseo e dentário (MACHA et al., 2017), no entanto, a desnutrição parece ter um efeito mínimo sobre o tempo de formação do dente, o que não ocorre em relação ao tecido ósseo. Estudo de Elamin e Liversidge (2013) comparou estágios individuais de desenvolvimento dentário entre grupos nutridos e desnutridos e demonstrou estabilidade do desenvolvimento da dentição em relação a outros sistemas biológicos, como o ósseo. Já outro estudo encontrou atraso tanto na idade óssea como na idade dentária em crianças com baixo peso (KUMAR et al., 2013).
Devido à infinidade de métodos existentes para avaliar idades óssea e dentária, é preciso distingui-los e optar por métodos amplamente utilizados e adequadamente ajustados à população em estudo. Nesse sentido, é preciso considerar a diferença entre crescimento e desenvolvimento esquelético, bem como entre desenvolvimento dentário e irrupção, pois, apesar de se relacionarem, são processos diferentes e que podem ser afetados de formas distintas frente a diferentes variáveis clínicas ou ambientais.

É importante também investigar outros aspectos quantitativos e qualitativos do desenvolvimento, mensurando alterações morfofuncionais, nutricionais e metabólicas. Em relação à $E B$, há estudos em andamento sobre efeitos da suplementação de vitamina $D$, considerando a baixa densidade mineral óssea como complicação sistêmica da doença (RODARI et al., 2017). Além disso, alterações no padrão de crescimento e desenvolvimento maxilomandibular e da oclusão vêm sendo relatadas, tais como atresia maxilar, apinhamento e impaç̧ões dentárias (NAVA; ÁNGELES; DURAN, 2014).

No estudo de Liversidge et al. (2005) foram avaliadas 44 crianças com EB distrófica recessiva com atraso na idade dentária não significativo no grupo. Assim como na presente amostra, ainda que não tenham sido mensurados parâmetros sistêmicos dos pacientes, eles apresentam subtipo grave, cujas características clínicas geram impacto na alimentação e na mobilidade. Avaliar parâmetros nutricionais e metabólicos poderiam ajudar a elucidar valores significativamente discrepantes entre a idade cronológica e dentária, ajudando a identificar fatores correlacionados a essas condições. Os resultados do presente estudo colaboram com a hipótese de que a avaliação do desenvolvimento dentário seja um parâmetro mais estável frente aos aspectos relacionados à condição clínica desses pacientes.

As mutações genéticas da EB distrófica comprometem o colágeno tipo VII, mas são os colágenos tipo I e III que se mostram envolvidos na formação dos dentes (ABRAHÃO et al., 2006). Também, o colágeno tipo l é o componente predominante do osso. Dessa forma, supõe-se que as possíveis alterações de desenvolvimento dentário e esquelético de pacientes com esse subtipo não tenham causa genética, mas seriam secundárias à EB pelas repercussões metabólicas sistêmicas e limitações funcionais. Destaca-se também que a ausência de espaço nas arcadas e a perda precoce de dentes decíduos, comuns nesses pacientes, podem gerar atrasos na irrupção e maloclusão dentária, ainda que o dente tenha se formado em tempo esperado.

Em relação à metodologia empregada, considerou-se meios amplamente utilizados e com boa correlação dos resultados (ESAN; YENGOPAL; SCHEPARTZ, 2017). O método de Demirjian foi originalmente aplicado em franco-canadenses e hoje é empregado em pesquisas de diversos diversos países (ESAN; YENGOPAL; SCHEPARTZ, 2017). Uma metanálise constatou bom desempenho dos métodos internacionais na população brasileira, sendo o 
método de Demirjian o mais prevalentemente empregado (FRANCO et al., 2020). Em adição, foi optado testar o método de Willems, por apresentar melhor precisão dos resultados, embora ambos os métodos apresentem tendência à superestimação (ESAN; YENGOPAL; SCHEPARTZ, 2017), tal como foi verificado no presente estudo. Vale notar uma limitação presente no método de Demirjian devido à ausência de valores convertidos em anos na tabela de pontuação para os estágios de cada dente. Isso contribuiu para que a idade dentária não variasse entre dois pacientes, cuja idade cronológica estava acima de 14 anos (Tabela 1). 0 mesmo não aconteceu utilizando-se o método de Willems, que fornece uma tabela com valores já convertidos em anos.

Ao considerar os valores individuais, observam-se pacientes com subestimação da idade (Tabela 1). Supõe-se que a superestimação, usualmente apresentada por esses métodos, mascara um possível grau de atraso no desenvolvimento dentário desses indivíduos, sobretudo dos pacientes que apresentaram resultados subestimados. Em relação aos achados de superestimação da idade dentária, não parece haver relação com a idade aparente que esses pacientes apresentam, pois a grande maioria apresenta idade aparente significantemente menor do que a idade cronológica, no que se refere, principalmente, à estatura.

Devido à extensão da faixa etária dos pacientes avaliados e aos métodos de predição de idade poderem apresentar diferentes resultados em diferentes grupos etários, sobretudo na infância e adolescência, em que há diferenças significantes decorrentes do crescimento e do desenvolvimento, a média isolada pode não ser um parâmetro confiável para se considerar. Para comparação entre estudos é importante considerar a estatística e os métodos de predição empregados, além das características da amostra.

Em relação às limitações deste estudo, por se tratar de uma doença rara, a amostra reduzida foi um fator restritivo para avaliar variáveis como faixa etária e sexo. Ainda houve dificuldades devido ao acesso reduzido dos pacientes nos serviços ambulatoriais, considerando as precauções necessárias em razão da pandemia pelo Covid-19, momento em que estava sendo realizado este estudo. É possível, futuramente, realizar estudos com meIhor amostragem e análise estatística. Percebe-se também a necessidade da realização de estudos sobre métodos de predição de idade dentária no Brasil, com aplicação de correções para ajustá-los à população estudada, visando a obter scores mais precisos. Vale ressaltar que, devido à diversidade étnica e à dimensão do território brasileiro, é necessário ainda considerar ajustes que qualifiquem os métodos aos grupos populacionais das diversas regiões do país, aumentando sua especificidade.

\section{CONCLUSÃO}

Neste grupo de pacientes com EB distrófica recessiva não houve divergência significativa entre as idades dentária e cronológica, com superestimação comum aos métodos utilizados. Sugere-se que o tempo de maturação dentária pode ser um parâmetro estável frente a fatores adversos ao desenvolvimento que são comuns a esse grupo.

A partir deste estudo, estimulam-se pesquisas sobre desenvolvimento dentário dos indivíduos portadores de $\mathrm{EB}$, com ampliação da amostragem e relacionando variáveis, tais como fatores nutricionais, socioeconômicos, comorbidades e desenvolvimento ósseo. Essas informações podem ser utilizadas para melhor entendimento das complicações associadas à EB e embasar cientificamente as ações de saúde para esse grupo, favorecendo o melhor planejamento das intervenções odontológicas, inclusive prevenção e redução do desenvolvimento ou severidade das alterações bucais. Também, as investigações sobre a idade biológica de pacientes com alterações sistêmicas podem contribuir para o campo da Odontologia Legal.

\section{REFERÊNCIAS}

ABRAHÃO, I. et al. Collagen analysis in human tooth germ papillae. Brazilian Dental Journal, Ribeirão Preto, v. 17, n. 3 p. 208-212, Feb. 2006.

BRASIL. Ministério da Saúde. Protocolo clínico e diretrizes terapêuticas (PCDT) para epidermólise bolhosa hereditaria e adquirida. Brasília, DF: 2019.

COHN, H. I.; TENG, J. M. C. Advancement in management of epidermolysis bullosa. Current Opinion in Pediatrics, Philadelphia, v. 28, n. 4, p. 507-516, Aug. 2016.

CONCEIÇÃO, E. L. N.; CARDOSO, H. F. V. Environmental effects on skeletal versus dental development ii: Further testing of a basic assumption in human osteological research. American Journal of Physical Anthropology, Philadelphia, v. 144, n. 3, p. 463-470, Mar. 2011.

COSTA, D. P. et al. Desnutrição energético-protéica e cárie dentária na primeira infância. Revista de Nutrição, Campinas, v. 23, n. 1, p. 119126, fev. 2010.

DEMIRJIAN, A.; GOLDSTEIN, H.; TANNER, J. M. A new system of dental age assessment. Human Biology, Detroit, v. 45, n. 2, p. 211-227, May. 1973.

DIMAISIP-NABUAB, J. et al. Nutritional status, dental caries and tooth eruption in children: a longitudinal study in Cambodia, Indonesia and Lao PDR. BMC Pediatrics, London, v. 18, n. 1, p. 300, Sept. 2018.

ELAMIN, F.; LIVERSIDGE, H. M. Malnutrition has no effect on the timing of human tooth formation. Plos ONE, San Francisco, v. 8, n. 8, p. e72274, Aug. 2013.

ESAN, T. A.; YENGOPAL, V.; SCHEPARTZ, L. A. The Demirjian versus the Willems method for dental age estimation in different populations: A meta-analysis of published studies. PLos ONE, San Francisco, v. 12, n. 11, Nov. 2017.

FRANCO, A. et al. Assessment of dental age estimation methods applied to Brazilian children: a systematic review and meta-analysis. Dentomaxillofacial Radiology, Tokyo, v. 50, n. 2, p. 20200128, June 2020.

KRÄMER, S. et al. Clinical practice guidelines: Oral health care for children and adults living with epidermolysis bullosa. Special Care in Dentistry, Chicago, v. 40, supl. 1, p. 3-81, Nov. 2020.

KUMAR, V. et al. The relationship between dental age, bone age and chronological age in underweight children. Journal of Pharmacy \& Bioallied Sciences, [s.I.], v. 5, n. supl, 1, p. S73 - 79, June 2013. 
LIVERSIDGE, H. M. et al. Epidermolysis bullosa and dental developmental age. International Journal of Paediatric Dentistry, Oxford, v. 15, n. 5, p.335-341, Sept. 2005.

MACHA, M. et al. Estimation of correlation between chronological age, skeletal age and dental age in children: A cross-sectional study. Journal of Clinical and Diagnostic Research: JCDR, [s.I.], v. 11, n. 9, p. ZCO1ZC04, Sept. 2017.

NAVA, E.; ÁNGELES, E.; DURAN, A. Stomatologic management of dental malocclusion in patients with dystrophic epidermolysis bullosa using an nterceptive guide of occlusion (IGO): Comparison of two cases. Revista Mexicana de Ortodoncia, [s.I.], v. 2, p. e112-e119, Apr. 2014.

PITTA, A. L.; MAGALHÃES, R. P.; SILVA, J. C. da. Epidermólise bolhosa congênita - importância do cuidado de enfermagem. CuidArte Enferm, Catanduva, v.10, n.2 p. 201-208, jul./dez. 2016.

RODARI, G. et al. Birmingham epidermolysis severity score and vitamin $D$ status are associated with low BMD in children with epidermolysis bullosa. Osteoporosis International, London, v. 28, n. 4 p. 1385-1392, Dec.2017.

WILLEMS, G. et al. Dental age estimation in Belgian children: Demirjian's Technique Revisited. Journal of Forensic Sciences, Philadelphia, v. 46, n. 4, p. 893-895, July 2001

ZIDORIO, A. P. C. et al. Nutritional aspects of children and adolescents with epidermolysis bullosa: literature review. Anais Brasileiros de Dermatologia, Rio de Janeiro, v. 90, n. 2, p. 217-223, Apr. 2015.
Submetido em: 19/11/2021

Aceito em: 20/11/2021 